\title{
CFD prediction of flutter of turbine blades and comparison with an experimental test case
}

\author{
Vaclav Slama ${ }^{1, *}$, Bartolomej Rudas ${ }^{1}$, Jiri $\mathrm{Ira}^{2}$, Ales Macalka ${ }^{2}$, Petr Eret $^{3}$, and Volodymyr \\ Tsymbalyuk ${ }^{3}$ \\ ${ }^{1}$ Doosan Skoda Power s.r.o., Tylova 1/57, Pilsen 30128, Czech Republic \\ ${ }^{2}$ NUM solution s.r.o., Perucká 2482/7, Prague 12000, Czech Republic \\ ${ }^{3}$ University of West Bohemia, Faculty of Mechanical Engineering, Department of Power System \\ Engineering, Univerzitní 8, Pilsen 30614, Czech Republic
}

\begin{abstract}
Last stage blades are a key element of steam turbines and in many ways determine the turbine configuration alongside with the overall turbine performance. The total efficiency of the low pressure turbine section can be increased by extending the last stage blades. The design process of such long blades involves a flutter analysis using CFD tools which have to be validated by measurements in test facilities under various operating conditions. Experimental data obtained from a subsonic wind tunnel with an oscillating turbine blade cascade, which is available at the Department of Power System Engineering at the University of West Bohemia, was compared with simulations in ANSYS CFX currently used in the Doosan Škoda Power. The paper provides a brief summary of experimental rig description, CFD tool setup and the results for the case of a travelling wave mode with the pure torsion motion of amplitude of $0.5^{\circ}, \mathrm{Ma}=0.2$, reduced frequency of 0.38 and angle of attack $+5^{\circ}$.
\end{abstract}

\section{Test rig description}

An in-draft wind tunnel which is shown in Figure 1 is utilized for experimental investigations of subsonic flutter in a blade cascade. Air enters to the system through a system of filters and turbulence reducing screens and then accelerates in a convergent nozzle before moving into a test section with a turbine blade cascade in a linear configuration which is shown in Figure 2. For the flutter investigation, this blade cascade consists of eight blades and the four central blades (3-6) are flexibly mounted and each has two degrees of freedom presenting bending and torsion motions. Because aerodynamic forces and moments are weak and can be supressed by blade inertial loading, the blades are made of carbon fibre. The construction of the test rig enables that the blade cascade can be adjusted for different angles of attack. In this paper, the case with the angle of attack $+5^{\circ}$ is presented.

Upstream of the cascade, there is a Pitot probe which is used for the steady flow inlet velocity measurement as well as the inlet static and the total pressure measurement.

* corresponding author: vaclav.slama@doosan.com 
Inlet flow static temperature was obtained using a thermometer sensor also situated in the upstream part. Downstream of the cascade, there is a traversing mechanism with a Pitotstatic probe in order to measure the pressure profiles downstream of the blade cascade. Before leaving the wind tunnel, the airflow discharges in the drum chamber and runs through the outlet duct towards the blower vacuum inlet.

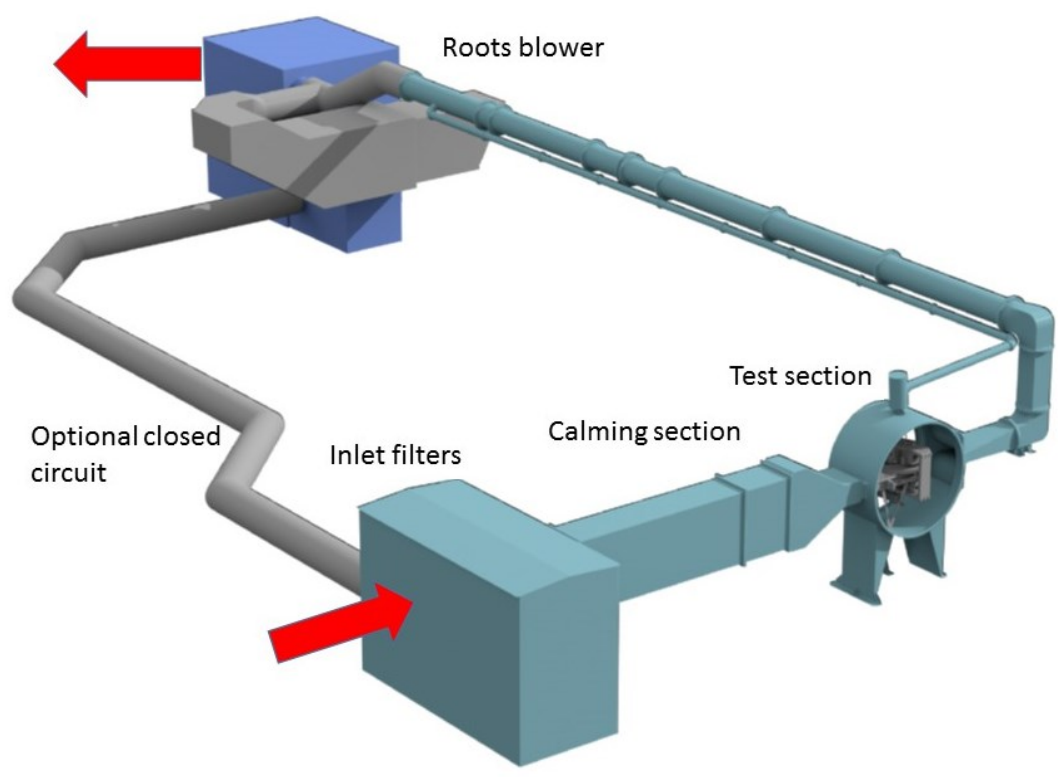

Fig. 1. The wind tunnel model.

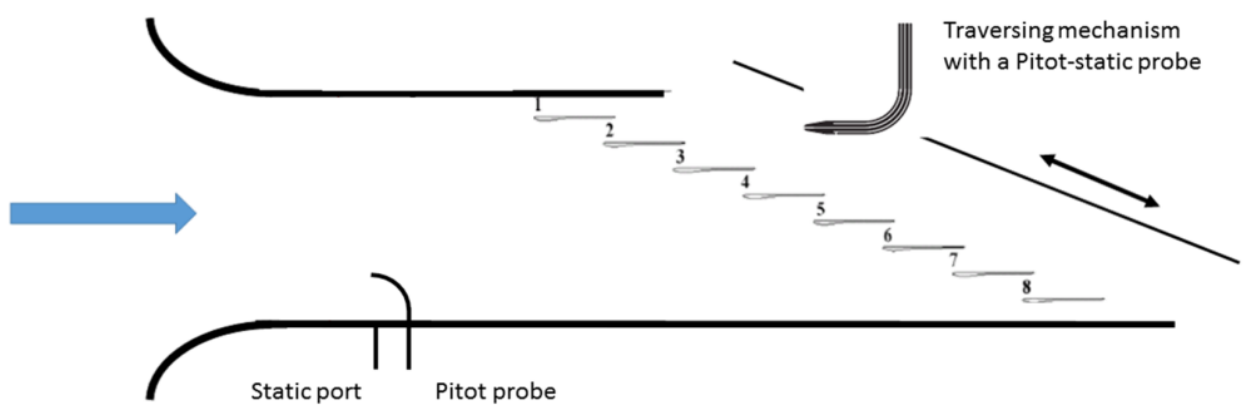

Fig. 2. Schematic of the test section, top view with the upstream probes, the linear blade cascade and the traversing plane.

The movements of central four blades (3-6) are controlled and simultaneously measured by four electromagnetic shakers, which are alternately attached at the top and bottom of a supporting frame as shown in Figure 3. From the measured signals the aerodynamic work per cycle done by the fluid on each moving blade can be calculated. Because flow has induced blade bending deformation during the measurement of the torsion motion, experimental data were corrected thus numerical simulations were performed on rigid blades [1]. 


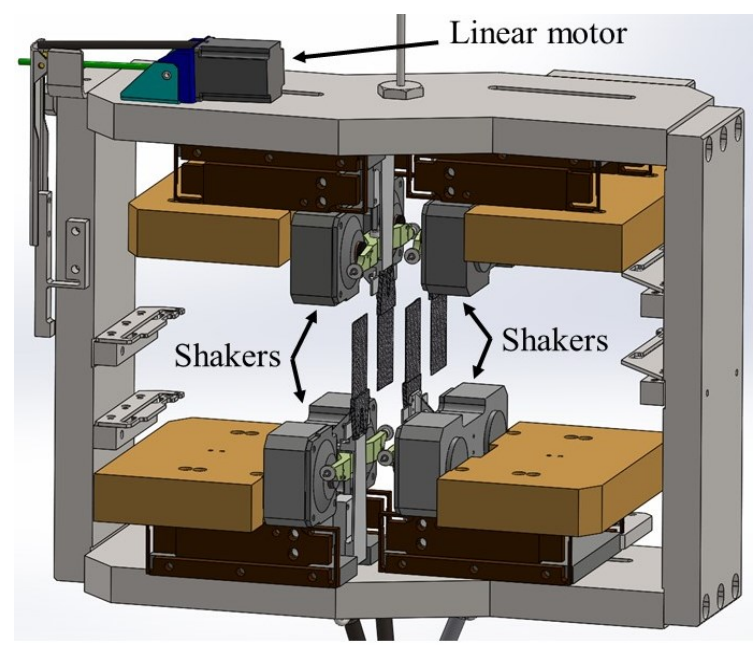

Fig. 3. Supporting frame with four electromagnetic shakers.

\section{Computational part}

A package of ANSYS 18.2 tools was used for performing CFD analyses. The numerical model was created for the angle of attack equal to $+5^{\circ}$. The static pressure, the total pressure and the static temperature in the measurement points upstream of the cascade were monitored during entire calculations and then their final values were compared with the experimental data. The position of this upstream measurement can be seen in Figure 2. The traverse plane downstream of the cascade, also depicted in Figure 2, was another important reference point. Static and total pressures at $50 \%$ of the blade height of the channel were monitored and their profiles were compared with the experimental data as well.

\subsection{Domains for numerical analysis and used mesh}

Before carrying out CFD calculations, it was necessary to simplify the 3D CAD model of the wind tunnel test section. Adjacent tunnel parts out of the main flow were removed and the model was separated into two sections (Figure 4). After several preliminary computations, the CFD model was further simplified and also improved (Figure. 5). Details about model simplifications and improvements can be found in [2].
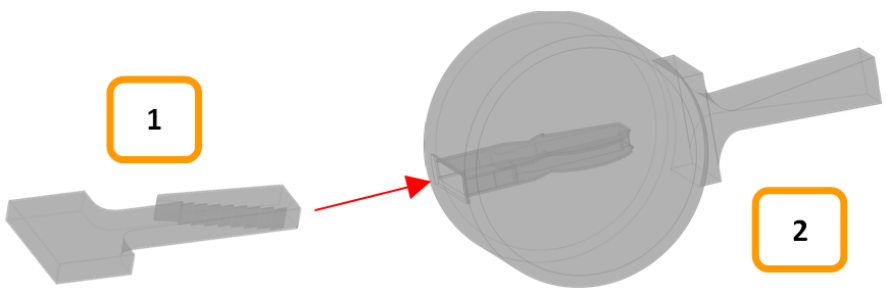

Fig. 4. Domain used during preliminary computations. 


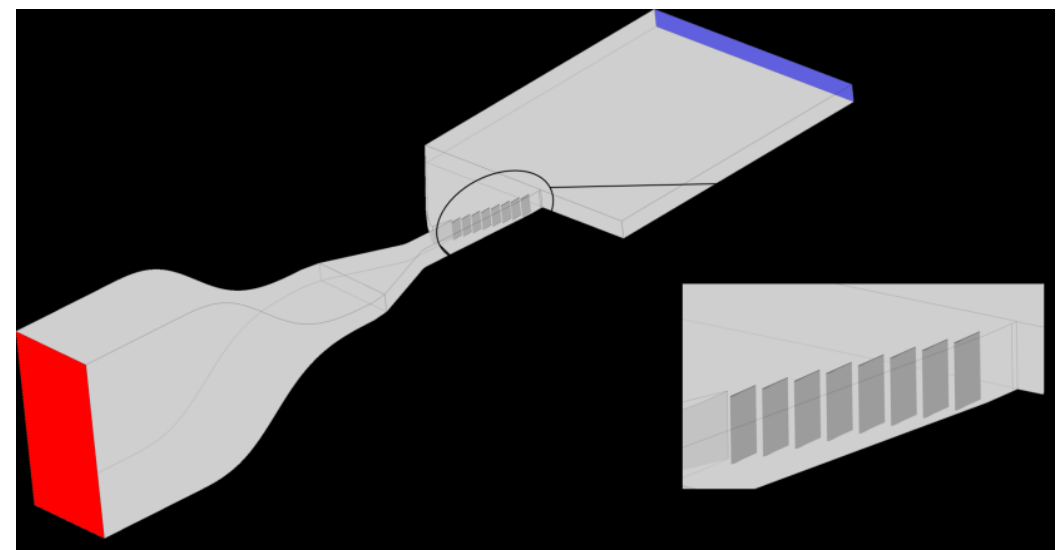

Fig. 5. Simplified and optimized domain with the detail of the blades.

A hexahedral mesh with an appropriate boundary layer, where the first element height is equal to $1.10^{-5} \mathrm{~m}$, on the blades and the adjacent walls was created for the whole computational domain in ANSYS ICEM CFD 18.2. The mesh which was used for steadystate simulations can be seen in Figure 6. There is also shown that, for unsteady simulations, the domain was reduced.

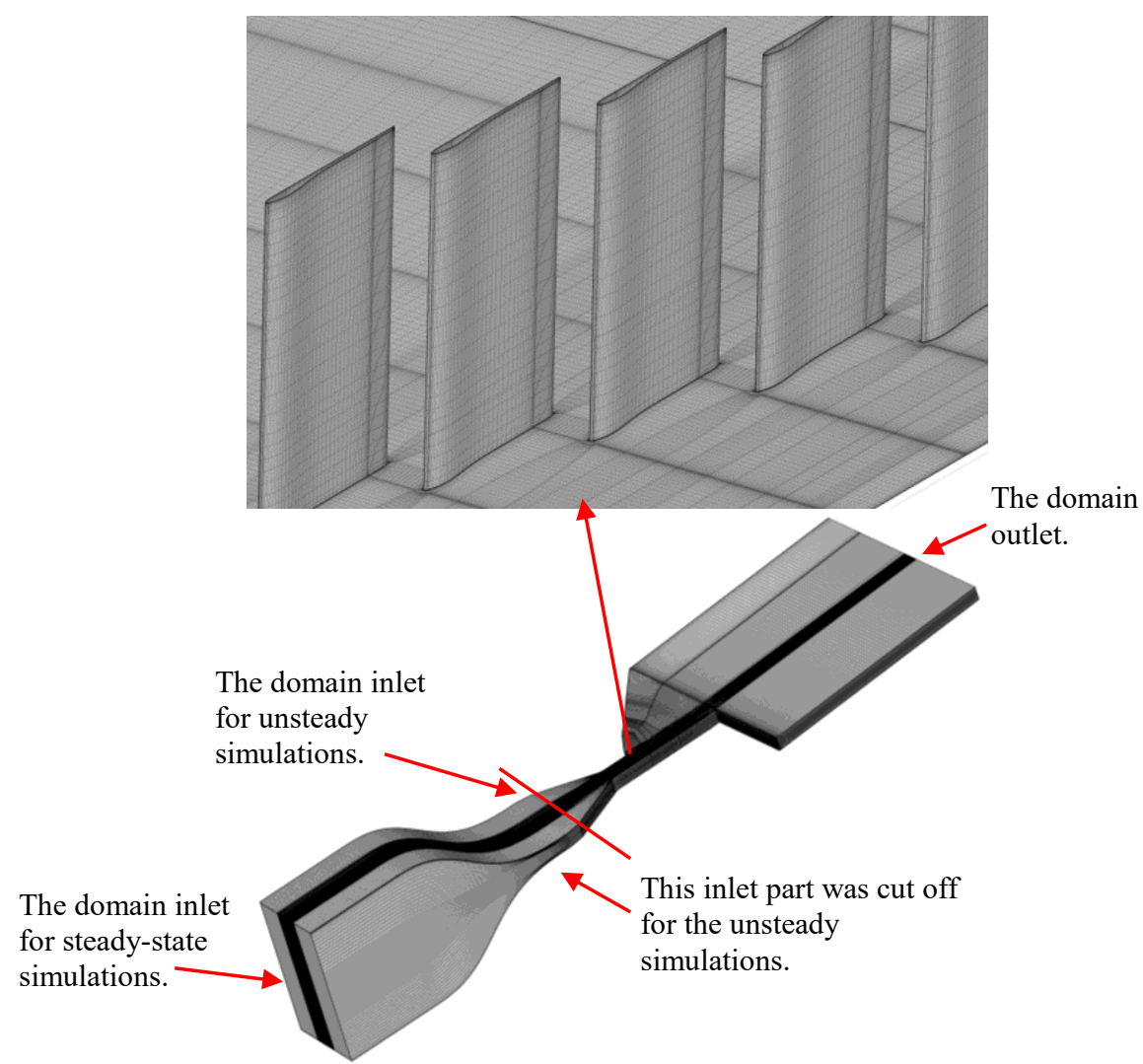

Fig. 6. Computational mesh for steady-state (16 million elements) and unsteady simulations (3.5 million elements) with the detail of blades. 


\subsection{Solver setup}

ANSYS CFX 18.2 was used as the main computational tool. For steady-state simulations, the flow field was modelled as steady, viscous and fully turbulent. The medium was defined as air. The ideal gas law was used to describe its thermodynamic properties. The two-equation eddy-viscosity SST k- $\omega$ turbulent model with an automatic wall function was used for turbulence modelling. Second order accurate approximations for all terms in the governing equations were applied.

For unsteady simulations, the basic settings were the same as for the steady-state simulations. Moreover, a time period was specified as the reciprocal value of the tuned frequency $(1 / 82.2 \mathrm{~Hz})$. The total number of time steps per period was chosen to be equal to 100 which corresponds to the time step $\Delta t=1.217 \cdot 10^{-4} \mathrm{~s}$. The total number of periods per run was set to 6 . The blade motion was defined according to the measurement as a pure torsion oscillation of amplitude of $0.5^{\circ}$ around the middle of the blade chord. The calculations were performed for full range of inter blade phase angles (IBPA $=0 \div$ $360^{\circ}$ ) with the step of $30^{\circ}$.

\subsection{Boundary conditions}

For steady-state simulations, the total pressure and the total temperature were defined at the domain inlet (see Figure 6). Using an iterative process, these inlet parameters were determined in such a way that the total pressure in the traverse plane downstream of the blade cascade was approximately equal to the measured values and the inlet static temperature was equal to the upstream measured value. The mass flow rate value was adjusted in a reasonable range so that the static pressure distribution in the traverse plain matches the experimental data. For unsteady simulations, the boundary conditions at the inlet of reduced domain (see Figure 6) and at the outlet were taken from the steady-state simulation results. A total pressure and a total temperature profile as well as velocity vectors were defined at the inlet and the mass flow rate was defined at the outlet.

\subsection{Steady-state CFD model validation}

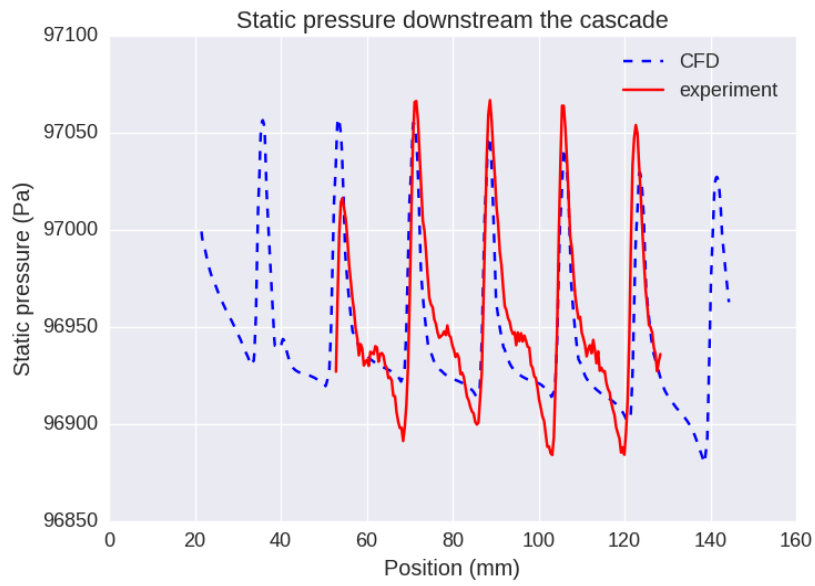

Fig. 7. Experimental and numerical results of static pressure in the traverse plane downstream of the cascade - see Figure 2) for defined conditions: $\mathrm{Ma}=0.2$, angle of attack $+5^{\circ}$. 


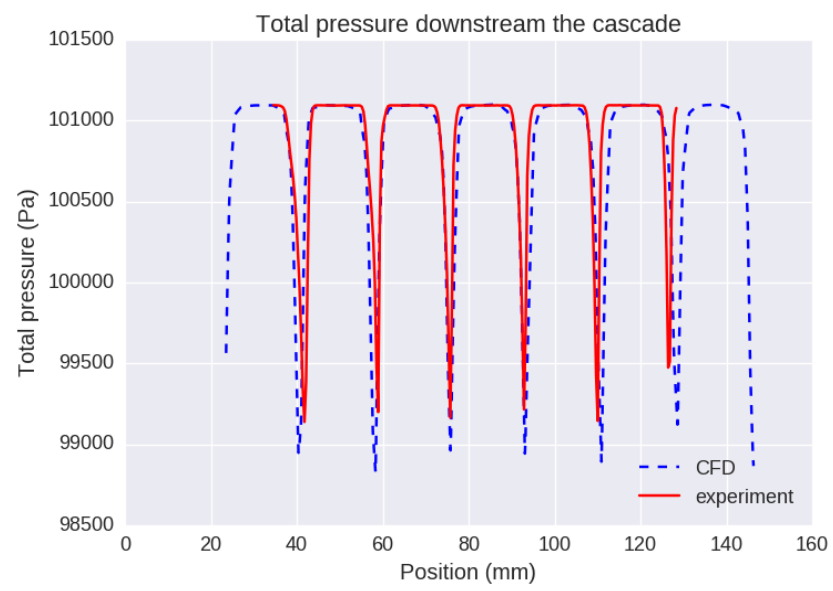

Fig. 8. Experimental and numerical results of total pressure in the traverse plane downstream of the cascade - see Figure 2) for defined conditions: $\mathrm{Ma}=0.2$, angle of attack $+5^{\circ}$.

A comparison between the experimental and numerical results of the total and static pressures in the traverse plane, which is situated downstream of the cascade, is depicted in Figures 7 and 8 . Both measurement and CDF simulation were carried out at $50 \%$ blade height. As expected, the numerical results correspond reasonably well with the experimental data. Several possible explanations of the difference between the results are presented in [2].

\section{Unsteady CFD and measurement comparison}

As a common indicator of the potential danger of flutter, the aerodynamic work in Eq. 1 is used. This work is defined as the work done by the fluid on the blade per vibration cycle:

$$
A W_{\text {cycle }}=\int_{t_{0}}^{t_{0}+T} \int_{A}-p \mathbf{v n} \mathrm{d} A \mathrm{~d} t
$$

where $T$ is the period of one vibration cycle, $t_{0}$ is the time at the start of the vibration cycle, $p$ is fluid pressure, $\mathbf{v}$ is the velocity of the blade caused by imposed vibrational displacement, $A$ is the surface of the blade and $\mathbf{n}$ is the surface unit normal vector. When the aerodynamic work per cycle done on the blade is positive, the blade is aerodynamically unstable (i.e. flutter). The aerodynamic work calculated during the unsteady numerical simulations is in excellent agreement with the experimental values as shown in Figures. 912. 


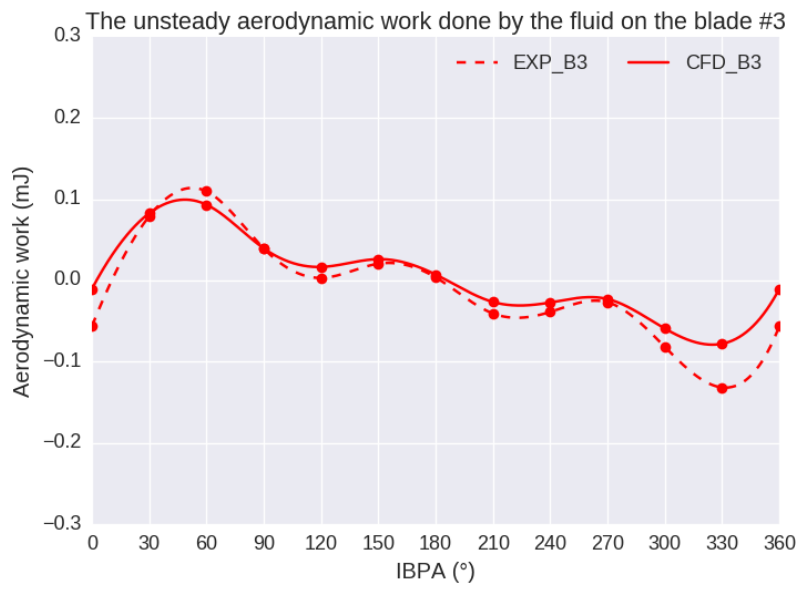

Fig. 9. Experimental (EXP) and numerical (CFD) results of the aerodynamic work done on blade \#3.

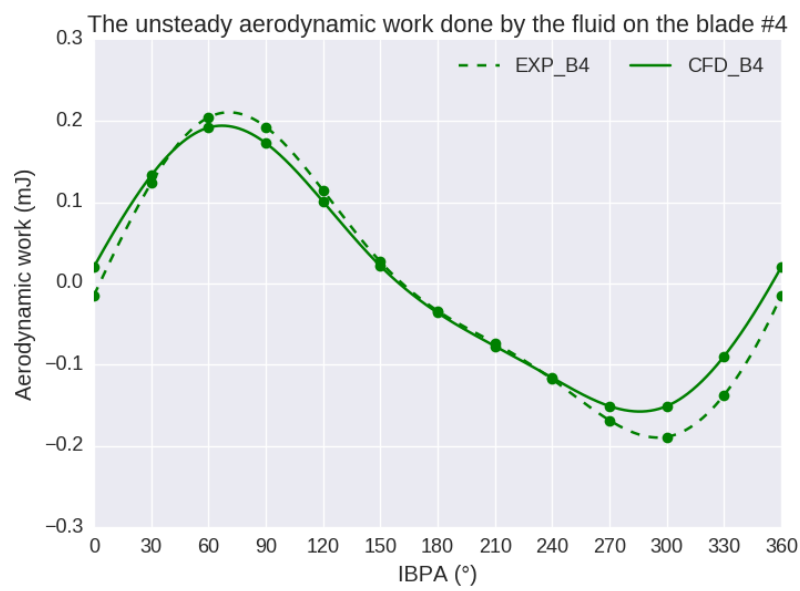

Fig. 10. Experimental (EXP) and numerical (CFD) results of the aerodynamic work done on blade \#4.

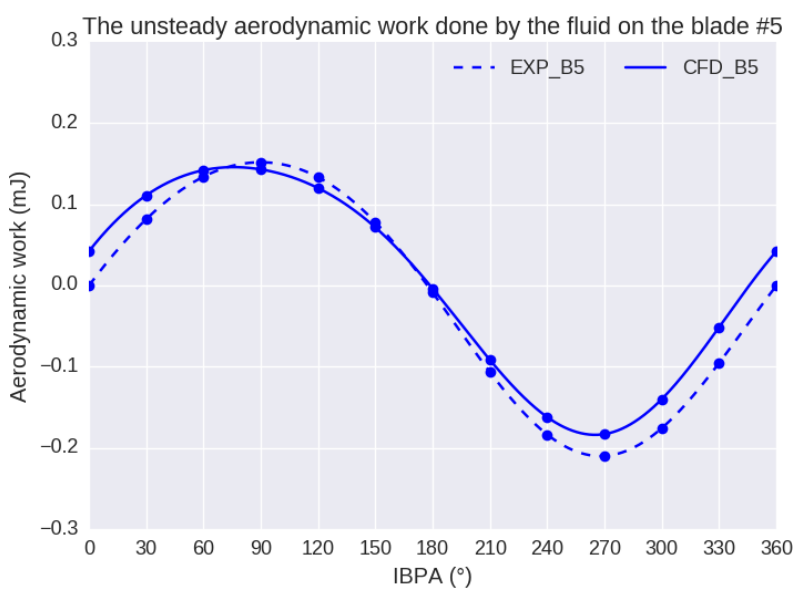

Fig. 11. Experimental (EXP) and numerical (CFD) results of the aerodynamic work done on blade $\#$. 


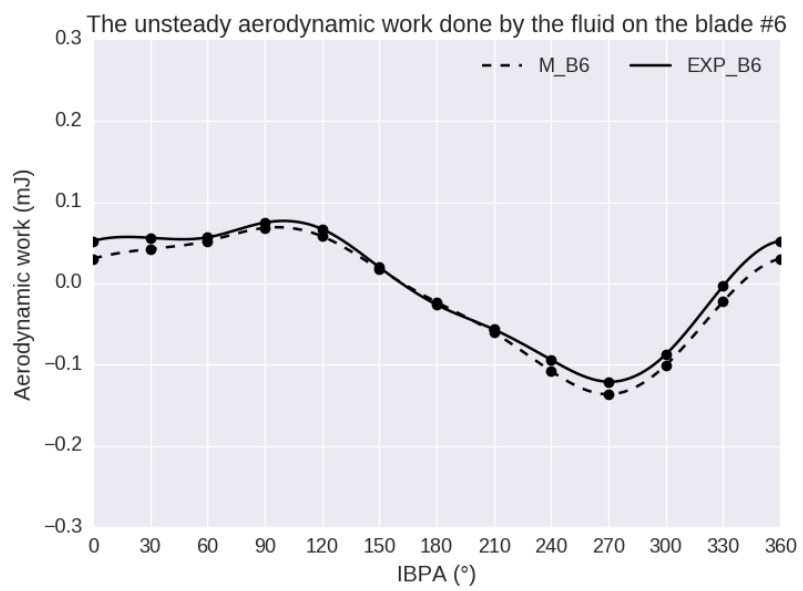

Fig. 12. Experimental (EXP) and numerical (CFD) results of the aerodynamic work done on blade \#6.

\section{Conclusions}

Aerodynamic work, which is an important parameter indicating the potential danger of blade cascade flutter, was evaluated from experimental measurements and CFD simulations for the case of a travelling wave mode with the pure torsion motion of amplitude of $0.5^{\circ}$, $\mathrm{Ma}=0.2$, reduced frequency of 0.38 and angle of attack $+5^{\circ}$. The experiments were carried out using an in-draft wind tunnel where a turbine blade cascade in a linear configuration with four flexibly mounted blades is installed. ANSYS CFX 18.2 was used to carry out the unsteady numerical analyses. In this test case, both CFD analysis and measurements showed excellent agreement. Further validation of flutter prediction and parametric study are necessary to verify dominant trends in flutter behaviour.

This research was founded by the European project Flexturbine, H2020 research and innovation programme under grant agreement No. 653941.

\section{References}

1. P. Eret, J. Ira, A. Macálka, B. Rudas, V. Sláma, V. Tsymbalyuk, ASME Turbo Expo, The Validation of Flutter Prediction in a Linear Cascade on Non-Rigid Turbine Blades, (to be published in 2018)

2. P. Eret, J. Ira, A. Macálka, V. Sláma, V. Tsymbalyuk, Experimental Fluid Mechanics, Experimental and CFD analysis of steady subsonic airflow in a stationary cascade of turbine blades, 230-234 (2017)

3. T. Tanuma, Woodhead Publishing, Advances in Steam Turbines for Modern Power Plants, Development of last-stage long blades for steam turbines, 13, 279-305 (2017)

4. K. Hourigan, F. Liu, I. McBean, M. Thompson, J. Fluids Eng., Prediction of Flutter of Turbine Blades in a Transonic Annular Cascade, 127-133 (2005) 